\title{
Editorial
}

\section{Signal Transduction Pathways in Chronic Inflammatory Rheumatic Diseases}

\author{
Andrew P. Cope $e^{*} \S$
}

\begin{abstract}
Academic Department of Rheumatology, Centre for Molecular and Cellular Biology of Inflammation, New Hunt's House, Guy's Campus, Great Maze Pond, School of Medicine, King's College London, London, SE1 1UL, UK
\end{abstract}

\section{INTRODUCTION}

The rheumatic diseases have become a test bed for new therapies in chronic inflammatory immune-mediated diseases - and rightly so. The inflammatory response is relatively easy to quantify, and validated outcome measures are available that permit investigation of efficacy over short periods of time. The late 1990s and early 2000s heralded the era of biological therapy, pioneered in diseases such as rheumatoid arthritis; clinical trials in inflammatory bowel disease and psoriasis followed soon after. These breakthroughs in medicine confirmed unambiguously that it was possible to suppress inflammation in vivo with highly specific, targeted therapy - in this case monoclonal antibodies or derivatives thereof. In the context of cytokine blockade it put to bed the notion that there was redundancy of cytokine cascades, implying that there were hierarchies, and that interfering with expression of inflammatory mediators at a proximal/early level was a rational approach to reduce the burden of these chronic disabling diseases.

As a result of these advances, there has been intense interest in addressing whether similar levels of suppression of inflammation could be achieved by interfering with the intracellular signal transduction pathways that underpin chronic inflammatory responses, in ways that are safe and which minimize off-target effects that might negate the therapeutic response or tolerability. There would be distinct advantages, not least in terms of cost, of administering orally active drugs that achieve similar response rates to the parenteral administered biologic agents. There are many pharmacologically tractable pathways to choose from. How does one prioritise these for further investigation?

It was with great anticipation that I invited some of the world's leading exponents in signal transduction to contribute to this series. I was not disappointed. The following reviews provide timely updates on progress in understanding both the wiring of such complex signaling networks, the impact of gene deletion and the potential of

*Address correspondence to this author at the Academic Department of Rheumatology, Centre for Molecular and Cellular Biology of Inflammation, New Hunt's House, Guy's Campus, Great Maze Pond, School of Medicine, King's College London, London, SE1 1UL, UK; Tel: +44 207848 6901; Fax: +44 207848 8632; E-mail: andrew.cope@kcl.ac.uk

${ }^{\S}$ Guest Editor specific signaling modules and their intermediates as valid targets for suppressing immune and inflammatory responses in man. While progress is more advanced with some pathways compared to others, studies where progress with pharmacological inhibition is relatively advanced have illustrated how the biology has directed approaches to manipulate signaling cascades for use in the clinical setting.

Okkenhaug and colleagues set the ball rolling with an elegant overview of how the PI3K signaling pathway evolved, how it becomes activated and how it's active components effects are regulated. Focusing on p110 $\gamma$ and p110 , the authors highlight the importance of understanding these intermediates in different cell types. Data from animal models of inflammation set the scene for cataloguing an array of perturbations of this pathway in rheumatoid arthritis (RA) and systemic lupus erythematosus (SLE). As regulators of cell activation, growth, proliferation, differentiation and survival, this pathway is a very attractive target for therapeutic manipulation.

The Ras family of small GTP binding proteins has been explored extensively in the context of cancer. An understanding of the mutations - both inherited and engineered - has provided major insights into understanding the biology of these signaling intermediates. Reedquist and Tak offer a tantalizing view of the complexities of the family of small GTPases, highlighting the parallels between invading fibroblast-like synoviocytes that underpin of cartilage destruction of synovial pannus in RA and invading tumour cells. Their conclusion that constitutive expression of $\mathrm{H}-\mathrm{Ras}$, combined with inflammation induced $\mathrm{K}$ - and N-Ras contributes substantially to this phenotype is particularly compelling. The role of Rap1 and its aberrant function in RA $\mathrm{T}$ cells is also described.

Janus kinase inhibitors are now in advanced stages of development for use in the treatment of immune-mediated inflammatory diseases. Indeed, one such inhibitor ruxolitinib, whose target is predominantly JAK1 and JAK2, is FDA approved for use in myelofibrosis, while tofacitinib has been tested extensively in immune-mediated diseases including RA, psoriasis and renal transplantation. This begs the question, how did the field get to this point? O'Shea, Laurence and colleagues provide some answers, elegantly summarising the biology of the JAK/STAT pathways that have underpinned these developments. Studies of genetargeted mice and the identification of patients with 
mutations in JAK family members have done much to highlight the importance of this kinase family. Perhaps the best recognized are the loss-of-function mutations of $J A K 3$ defining a subgroup of SCID patients, and the gain-offunction mutations of $J A K 2$ responsible for a number of lymphoproliferative and myeloproliferative diseases. That these pathways are of particular importance in $\mathrm{T}$ cell biology, serving to skew pathways of differentiation along distinct lineages, means that the capacity to manipulate these pathways, particularly those relevant to the development and maintenance of Th17 effector T cells, is therefore of high relevance to autoimmune disease.

Clark and Dean summarise compelling evidence to support the notion that p38 MAPK pathway plays crucial roles in regulating inflammatory gene expression. Recent advances indicate that the initiation of activation of $\mathrm{p} 38$ MAPK pathway involves the recruitment and activation of ubiquitin ligases - an emerging concept relevant to a growing number of signal transduction pathways, besides NF- $\kappa \mathrm{B}$. Notable are the contributions that p38 biology have made to understanding regulation of mRNA stability and degradation, and the induction of negative regulatory pathways, including MAPK phosphatases, some of which are induced by glucocorticoids. Initial attempts to block this pathway in the clinic were fraught with unexpected adverse events, but a more detailed understanding of the mechanisms by which p38 regulates gene expression and a posttranscriptional level has led to the identification of downstream targets that might provide more selective ways to manipulate this pathway and its downstream effects.
Another stress kinase, the c-Jun N-terminal kinase, has featured widely as an attractive target in inflammation in the last two decades, not least because of it is activated by a wide range of environmental cues including tumour necrosis factor $\alpha$ and interleukin-1. The Jnk family are encoded by three genes, but alternative splicing generates at least 10 gene products, adding further complexity to the biology of this kinase. Firestein elaborates on how these gene products are activated through a cascade of phosphorylation events, coordinated by upstream multiple MAP kinase kinase kinases (MKKKs) and dual specificity MAPKK, notably MKK4 and MKK7, and, like p38 MAPK, regulated by a family of MAPK phosphatases. This elegant review also provides compelling evidence for the concept of isoform specific gene functions. Particularly relevant to rheumatic diseases are the target genes of Jnk activation and its associated transcription factors downstream, including matrix metalloproteinases (MMP), notably MMP3 and MMP13. Gene deletion and Jnk inhibition studies provide ample in vivo and in vitro evidence of tissue protection, impacting in addition on pathways of cell migration, differentiation and survival. Selective Jnk inhibition remains an exciting prospect.

This series provides something for readers from a wide range of disciplines, with a wide range of interests, including basic molecular and cellular biologists, as well as those clinician scientists considering future prospects for orallyactive small molecule inhibitors of inflammatory pathways. I sincerely hope you enjoy this set of outstanding reviews as much as I have.

(C) Andrew P. Cope; Licensee Bentham Open.

This is an open access article licensed under the terms of the Creative Commons Attribution Non-Commercial License (http://creativecommons.org/licenses/by-nc/ 3.0/) which permits unrestricted, non-commercial use, distribution and reproduction in any medium, provided the work is properly cited. 